\title{
PRIMARY INTERSECTIONS FOR TWO SIDED IDEALS OF A NOETHERIAN MATRIX RING
}

\author{
BY \\ EDMUND H. FELLER
}

Introduction. The purpose of this paper is to obtain certain primary intersections as described in [1] for all two sided ideals in the matrix ring $D_{n}$ where $D$ is a Noetherian ring. We refer to such a ring as a Noetherian matrix ring. The primary intersections will depend only upon the Noetherian ring $D$. The following discussion will show that if the primary intersections of the ideals in $D$ are known one can immediately write primary intersections for all two sided ideals in $D_{n}$.

1. Reformulation of theorems. The following two theorems are reformulations of the author's Theorems 2.5 and 2.7 of [1]. The proofs are very similar to those of Noether [5] and Krull [4] and therefore were not included in [1] and are not included here. (See [2, pp. 172-181]). The definitions of [1] are used here.

Theorem 1.1. Let $N=N_{1} \cap \cdots \cap N_{s}=N_{1}^{\sharp} \cap \cdots \cap N_{s}^{\sharp}$ be irredundant intersections where $N_{i}, N_{i}^{*}$ are irreducible $R$ submodules, $i=1,2, \cdots, s$. Let $H$ be a subring of $\bigcap_{i=1}^{s}\left[V^{*}\left(N_{i}\right) \cap V^{*}\left(N_{i}^{\sharp}\right)\right]$ containing the identity element, then the set of distinct $H$ radicals of $N_{1}, \cdots, N_{s}$ is identical with the set of distinct $H$ radicals of $N_{1}^{\#}, \cdots, N_{s}^{\#}$ in $H$.

From this theorem and Theorem 2.6 of [1] we have

Theorem 1.2. Let $N$ be an $R$ submodule of the $A-R$ module $M$ which satisfies the A.C.C. for $R$ submodules $\left({ }^{1}\right)$. Let $\alpha$ be an index that ranges over a possibly infinite set $G$ whose cardinal number is $\psi$ and let $N=\bigcap_{i=1}^{t} N_{i \alpha}$ be a set of $\psi$ irredundant representations of $N$ as the intersection of irreducible $R$ submodules $N_{i \alpha}$ of $M$. Let $H(G)$ be a subring with identity of $\cap V^{*}\left(N_{i \alpha}\right)$ where $i$ ranges from 1 to $t$ and $\alpha$ ranges over $G$. Then for the $\alpha$ th intersection there exist $H$ primary $R$ submodules $N_{1 \alpha}^{\prime}, \cdots, N_{s \alpha}^{\prime}$ with distinct $H$ radicals $p_{1}, \cdots, p_{s}$ such that $N$ $=N_{1 \alpha}^{\prime} \cap \cdots \cap N_{s \alpha}^{\prime}$. If $N=N_{1 \beta}^{\prime} \cap \cdots \cap N_{r \beta}^{\prime}$ is another such intersection where $\beta$ is an index of $G$ then $r=s$ and for a suitable rearrangement of the subscripts the corresponding $H$ radicals are equal.

2. Primary intersections for two sided ideals in $D_{n}$. A ring with identity as a A-R module if one takes as $A$ the ring of left multiplications and as $R$ the ring of right multiplications. Thus theorems of this paper and [1] apply to rings with identity that satisfy the A.C.C. for right ideals.

Received by the editors April 22, 1957.

(1) An A-R module is defined as a right A, right $\mathrm{R}$ module in [3, page 17]. 
Let $D$ be a Noetherian ring. We shall consider the application of these theorems to all two sided ideals of $D_{n}$, the ring of $n$ by $n$ matrices with elements in $D$.

If $I$ is an ideal of $D$ then the set of all matrices $\left(a_{i j}\right)$ with $a_{i j} \in D$ for $i \neq k$ and $a_{k j} \in I$ is a right ideal of $D_{n}$ which we shall denote by $(I, k)$.

Statement 2.1. If $I$ is an ideal of $D$ and $(I, k)$ is contained in a right ideal $H$ of $D_{n}$ then $H$ is of the form $\left(I^{\prime}, k\right)$ where $I^{\prime}$ is an ideal of $D$ which contains $I$.

Proof. Suppose as in the statement that $(I, k) \subseteq H$. The set of elements $I^{\prime}$ that appear in the $k$ th row of $H$ is an ideal of $D$. For suppose $a, b \in I^{\prime}$ and say $a$ appears in the matrix $A$ of $H$ in the $(k, i)$ position and $b$ appears in the matrix $B$ of $H$ in the $(k, j)$ position. Let $E_{i j}$ denote the matrix with 1 in the $(i, j)$ position and zero elsewhere. Then $A E_{i 1}+B E_{j 1}$ is a matrix which contains $a+b$ in the $k$ th row. If $c \in D$ then $A E_{i 1} c$ is a matrix which contains $a c$ in the $k$ th row. Next we shall show that if $c$ is an element of $I^{\prime}$ then $H$ contains a matrix with $c$ in position $(k, 1)$ and zero elsewhere. Since $c \in I^{\prime}$ there exist a matrix with $c$ in the $k$ th row and by proper multiplication by the elements of $D_{n}$ on the right $H$ must contain a matrix $\left(a_{i j}\right), a_{i 1} \in D, a_{k 1}=c$, $a_{i j}=0$ for $j>1$. Since $(I, k) \subseteq H, H$ contains a matrix $\left(b_{i j}\right), b_{i 1}=a_{i 1}, b_{k 1}=0$, $b_{i j}=0$ for $j>1$. Hence $\left(a_{i j}\right)-\left(b_{i j}\right)=\left(c_{i j}\right)$ where $c_{1 k}=c, c_{i j}=0$ for $i \neq 1$ and $j \neq k$. Consequently, since the right ideal $(0, k) \subseteq(I, k) \subseteq H$, we have $\left(c_{i j}\right)+(0, k)$ $\subseteq H$, i.e., the element $c$ appears in the $k$ th row of the first column and hence in every column with all combinations of the elements of $D$ in the $j \neq k$ rows. Since this is true for all elements $c \in I^{\prime}$, we have $H=\left(I^{\prime}, k\right)$.

Statement 2.2. If $I$ is an irreducible $\left({ }^{2}\right)$ ideal of $D$, then $(I, k), k$ $=1,2, \cdots, n$, is an irreducible $\left({ }^{2}\right)$ right ideal of $D_{n}$.

Proof. We shall prove that $(I, k)$ is irreducible. Suppose $(I, k)=H_{1} \cap H_{2}$ where $H_{1}$ and $H_{2}$ are right ideals of $D_{n}$ which properly include $(I, k)$. Then by Statement 2.1, $H_{1}$ and $H_{2}$ are of the form $\left(I_{1}, k\right)$ and $\left(I_{2}, k\right)$, where $I_{1}$ and $I_{2}$ are ideals of $D$. Hence $I=I_{1} \cap I_{2}$ where $I_{1}$ and $I_{2}$ properly include $I$-contradiction.

For an irreducible ideal $I$ of $D$ we have from [1, Theorem 2.1] and the preceding statement that $(I, k)$ is $V^{*}[(I, k)]$ primary, where $V^{*}[(I, k)]$ is the set of elements $A$ in $D_{n}$ such that $A(I, k) \subseteq(I, k)$, in the sense that if $A B \in(I, k), B \in(I, k), A \in V^{*}[(I, k)]$, then $A^{t} \in(I, k)$ for some positive integer $t$.

Let $\left(I_{1}, I_{2}, k\right)$ denote the set of all matrices $\left(a_{i j}\right)$ where $a_{i j} \in D, i \neq k$, $a_{k j} \in I_{1}, j \neq k$, and $a_{k k} \in I_{2}$ where $I_{1}$ and $I_{2}$ are ideals of $D$.

Statement 2.3. $V^{*}[(I, k)]=(I, D, k)$.

Proof. Certainly $(I, D, k) \subseteq V^{*}[(I, k)]$. Suppose $A=\left(a_{i j}\right) \notin(I, D, k)$, say $a_{k j} \notin I$ for $j \neq k$. Let $E_{j k}$ denote the matrix with 1 in the $(j, k)$ position and zero

(2) An ideal here is irreducible in the sense that it is not the intersection of two right ideals which properly contain it. 
elsewhere. Then for $E_{j k}$ contained in $(I, k)$ we have $A E_{j k} \notin(I, k)$ since $A E_{j k}$ contains $a_{k j}$ in the $(k, k)$ position. This proves the statement.

The $V^{*}$ radical of $(I, k)$ in $(I, D, k)$ is the set of matrices $A$ in $(I, D, k)$ such that $A^{t} \in(I, k)$ for some positive integer $t$.

Statement 2.4. The $V^{*}$ radical of $(I, k)$ is $(I, P, k)$ where $P$ is the radical of the ideal $I$ in $D .(I, P, k)$ is a completely prime two sided ideal of $V^{*}$.

Proof. If $\left(a_{i j}\right) \in(I, D, k)$, then $\left(a_{i j}\right)^{t}=\left(b_{i j}\right)$ where $b_{i j} \in D, i \neq k, b_{k j} \in I$, $j \neq k, b_{k k}=c+a_{k k}^{t}$ for $c \in I$. Hence $\left(a_{i j}\right)^{t}$ is contained in $(I, k)$ if and only if $a_{k k}^{t} \in I$ for some positive integer $t$, i.e., $a_{k k}$ is contained in the radical of $I$ in $D$. The second part follows from Theorem 2.2 of [1].

From [3, p. 40] the ideals of $D_{n}$ are of the form $I_{n}$ where $I$ is an ideal of $D$. From the irreducible intersections for $I$ in $D$ we can write irreducible intersections for $I_{n}$ in $D_{n}$. This is displayed in the next theorem the proof of which is most direct and is therefore omitted.

Theorem 2.1. Let $I_{n}$ be a two sided ideal in $D_{n}$ where $D$ is a Noetherian ring. Let $\alpha$ be an index that ranges over a possibly infinite set $E$ whose cardinal number is $\sigma$ and let $I=I_{1 \alpha} \cap I_{2 \alpha} \cap \cdots \cap I_{s \alpha}$ be a set of $\sigma$ irredundant representations of $I$ as an intersection of irreducible ideals $I_{i \alpha}$ in $D$. Then the equation

$$
I_{n}=\bigcap_{i=1}^{s} \bigcap_{k=1}^{n}\left(I_{i \alpha_{k}}, k\right)
$$

in which, for each value of $k, a_{k}$ is an arbitrary index from the set $E$, defines $\sigma^{n}$ representations of $I_{n}$ as an irredundant intersection of irreducible right ideals of $D_{n}$.

For an intersection of the form $(Z)$, since the $V^{*}$ radical of $\left(I_{i \alpha_{k}}, k\right)$ is $\left(I_{i \alpha_{k}}, P, k\right)$ where $P$ is the radical of $I_{i \alpha_{k}}$, these radicals will all be different. In addition if different ideals of $I$ are used in two intersections for $I_{n}$ of the form $(Z)$ none of the $V^{*}$ radicals will be equal.

Let us now apply Theorems 2.6 of [1], 1.1, and 1.2 of this paper. For the $\sigma$ intersections of Theorem 2.1, consider as in Theorem 2.1 the set $S$ of $\sigma^{n}$ intersections for $I_{n}$ which can be formed from this set. Then $H=\cap V^{*}\left(I_{i \alpha}, k\right)$ where this intersection is taken over all $i, k$, and $\alpha$, which of course could be an infinite intersection. However for any one intersection of the form $(Z)$ the intersection of the $n V^{*}\left(I_{i \alpha}, k\right)$ 's involved in this intersection is the set of all matrices $\left(a_{i j}\right)$ where $a_{i j} \in I$ for $i \neq j, a_{i i} \in D$ which we shall denote by $(I \backslash D)$. Since this is true for all intersections of the form $(Z)$ we have $H=(I \backslash D)$ which is a finite intersection. In general if $I_{1}$ and $I_{2}$ are ideals of $D$ we shall denote by $\left(I_{1} \backslash I_{2}\right)$ the set of all matrices $\left(a_{i j}\right)$ with $a_{i j} \in I_{1}$ for $i \neq j, a_{i i} \in I_{2}$. Then the radical of $\left(I_{i \alpha}, k\right)$ in $(I \backslash D)$ will be the set of all matrices $\left(a_{i j}\right)$ where $a_{i j} \in I, i \neq j, a_{i i} \in D$ for $i \neq k, a_{k k} \in P$ where $P$ is the radical of $I_{i \alpha}$ in $D$. We 
shall denote such an ideal by $(I \backslash D, P, k)\left({ }^{3}\right)$. Consequently in $(I \backslash D)$ two radicals $\left(I \backslash D, P_{1}, k_{1}\right)$ and $\left(I \backslash D, P_{2}, k_{2}\right)$ will be equal if and only if $P_{1}=P_{2}$ and $k_{1}=k_{2}$. Thus we can apply Theorem 2.6 of [1] and combine the right ideals of $(Z)$ which have the same radicals in $(I \backslash D)$. This will result in an intersection of primary $(I \backslash D)$ right ideals of $D_{n}$ which is equal to $I_{n}$.

Since two radicals $\left(I \backslash D, P_{1}, k\right)$ and $\left(I \backslash D, P_{2}, m\right)$ will be equal if and only if $P_{1}=P_{2}$ and $k=m$ then in $(Z)$ we have $\left(I_{i \alpha_{k}}, k\right) \cap\left(I_{j \alpha_{m}}, m\right)$ will be $(I \backslash D)$ primary if and only if $k=m$ and $I_{i \alpha_{k}} \cap I_{j \alpha_{m}}$ is a primary ideal of $D$. Thus in applying Theorem 2.6 of [1] we combine the right ideals of $(Z)$ to write $(I \backslash D)$ primary intersections for $I_{n}$ with distinct $(I \backslash D)$ radicals.

From the previous discussion and Theorem 1.2 we have

Theorem 2.2. Let $I_{n}$ be a two sided ideal of $D_{n}$ where $D$ is a Noetherian ring. Let $\alpha$ be an index that ranges over a possibly infinite set $F$ whose cardinal number if $\rho$ and let $I=J_{1 \alpha} \cap \cdots \cap J_{r \alpha}$ be a set of $\rho$ intersections for $I$ where $J_{i \alpha}$ are primary ideals of $D$. Then the equation

$$
I_{n}=\bigcap_{i=1}^{r} \bigcap_{k=1}^{n}\left(J_{i \alpha_{k}}, k\right)
$$

in which, for each value of $k, \alpha_{k}$ is an arbitrary index from the set $F$, defines $\rho^{n}$ representations of $I_{n}$ as the intersection of $(I \backslash D)$ primary right ideals where the $(I \backslash D)$ radicals are distinct for each representation. For any two of these representations the $(I \backslash D)$ radicals are for some ordering equal.

One may now ask: (1) Are the primary intersections for $I_{n}$ discussed here the only such intersections? (2) How can one write primary intersections for all right ideals of $D_{n}$ ?

\section{BIBLIOGRAPHY}

1. E. H. Feller, The lattice of submodules of a module over a noncommutative ring, Trans. Amer. Math. Soc. vol. 81 (1956) pp. 342-357.

2. N. Jacobson, Lectures in abstract algebra, Van Nostrand Co., 1951.

3. - Structure of rings, Amer. Math. Soc. Colloquium Publications, vol. 37, 1956.

4. W. Krull, Ein neuer Beweis für die Haupsätze der allgemeinen Idealtheorie, Math. Ann. vol. 90 (1923) p. 55.

5. E. Noether, Idealtheorie in Ringbereichen, Math. Ann. vol. 83 (1921) pp. 24-66.

UNIVERSITY OF Wisconsin-MilWAUKEe, MilwaUkee, Wis.

(3) The radical of $I$ in $(I \backslash D)$ will be equal to $(I \backslash P)$ where $P$ is the radical of $I$ in $D$. 